\title{
Education and leadership
}

\section{Learning from the Integrated Care Pilot in West London}

\author{
Samantha Roberts MB BCh MBA \\ External Consultant \\ Neha Unadkat BSc (Hons) MSc MBA \\ Integrated Care Pilot Project Manager
}

Raj Chandok MBBS MRCGP DRCOG DC DFFP

Principal and Vice Chair Ealing Clinical Commissioning Group, Chair Ealing Integrated Care Pilot

Thirza Sawtell RN RHV BA (Hons) MHM

Director of Strategy and Service Transformation, NHS North West London

\section{Key messages}

We implemented a system to integrate care for patients with diabetes and/or over the age of 75 in an urban area with a population of 1.2 million. Within five months, all the acute, mental health and community trusts, and all the local authorities had signed up to participate, along with $84 \%$ of GP practices in the area. Patients and practitioners found the system of benefit in improving communication and collaboration.

- The first phase of the Outer North West London Integrated Care Pilot (ONWL ICP) has shown that it is possible to implement large-scale, multi-agency change in a short time to enable coordinated care for patients with long-term conditions.

- The second phase needs to more purposefully integrate with local programmes of work, including training the chairs of the monthly case conferences as leaders of transformational change who can lead annual cycles of interorganisational continuous quality improvement.

\section{Why this matters to us}

We are clinicians and managers who have been closely involved in the planning and implementation of the integrated care system described here. We feel that current systems are fragmented and do not support delivery of holistic, coordinated care. We are convinced of the benefits of multiple agencies working together to share their expertise of patients' social, psychological and physical needs.

Keywords: integrated care, long term conditions

\section{Background}

Throughout North West London policymakers recognised that an ageing population with an increasing burden of long-term conditions was leading to a rise in unplanned admissions. In 2011, this led three of the boroughs in Inner North West London (INWL) to develop an approach called the Integrated Care Pilot 
(ICP). ${ }^{1,2}$ Chairs of the Clinical Commissioning Groups in the four boroughs of the Outer North West London (ONWL) sector reviewed learning from the first year of the ICP and agreed to adopt a modified version. Such systems around the world had improved care, but typically took years to implement. However, the financial pressures we faced were imminent, making it important to implement any potential solution rapidly.

The aims of the Outer North West London Integrated Care Pilot (ONWL ICP) were to improve outcomes and reduce costs for patients with complex needs by taking a coordinated and proactive approach to their care. Following the model pioneered in Inner North West London, ${ }^{1,2}$ the ONWL ICP was arranged around multidisciplinary groups (MDGs) which covered a population of 50,000 (termed 'health networks'). Participants include GPs, acute consultants, mental health consultants, social workers, district nurses and specialist nurses. Our approach involved five key components:

- identifying patients with the most complex health and social care needs: using PARR++ and READ code searches;

- developing holistic pathways that describe a common, best practice approach to managing diabetic and elderly patients;

- co-creating care plans with patients with the most complex needs using bespoke EMIS and Vision IT templates;

- holding meetings of MDGs, centred around discussion of complex cases, twice a month;

- developing innovative service improvements, funded non-recurrently.

\section{Setting}

ONWL ICP was launched in the Boroughs of Brent, Ealing, Harrow and Hillingdon, an urban area with a population of 1.2 million. It involved collaborative working among primary care, acute hospitals, mental health and community trusts, social care and patient charities. The patient groups targeted were adults with diabetes and/or over the age of 75 .

\section{Method}

A project board was set up in May 2012 to engage commissioners, clinicians, social care and other stakeholders and to plan the pilot. In August 2012, the ONWL ICP was officially launched. It had an operational team, an independently chaired management board, four integrated management groups each co-chaired by a GP and a representative of social care, and 20 MDGs.

Clinical and social care leaders supported by an operational team:

- reviewed the international and local experience;

- contacted stakeholders and presented the case for change;

- identified clinical champions and leaders;

- secured resources to kick-start the intervention;

- provided the drive, administrative support and system for data collection;

- set up a structure for shared governance;

- supported the development of multidisciplinary groups, between three and six in each borough; and

- agreed care protocols and pathways.

\section{Role of MDGs}

- Held case conference.

- Agreed to review their performance quarterly.

\section{Role of member general practices}

- Agreed to create individual patient care plans, detailing interventions, that could be shared across settings of care.

- Selected complex cases to discuss at MDG meetings.

To evaluate progress, in November 2012 we carried out a questionnaire survey of 144 MDG members and 25 patients who had completed care plans in ONWL. We also interviewed 12 patients and facilitated discussions at 14 MDG meetings. The findings are summarised below.

\section{Results}

By December 2012, 767 care plans had been completed, 20 MDGs had been set up, and 180 case conferences had been held, discussing 798 patients. These results demonstrated that the activities intended to support integrated care were being carried out.

\section{Impact on patients and practice}

- The ICP processes helped general practices to develop a more proactive approach to patients with long-term conditions:

- $62 \%$ of GPs and practice nurses surveyed reported that the ICP has changed the way their practices work. In particular, their practices had 
developed increased emphasis on identifying high-risk patients and proactively managing them.

- Patients found the experience of care planning to be valuable. Care planning sessions were well received by patients:

- $100 \%$ of patients said that they discussed what was most important to them in managing their own health;

- $96 \%$ of patients thought that the care planning discussion would improve their own efforts at self-management;

- $68 \%$ of patients said that they received goodquality information during the session.

- Productive relationships were built across organisational boundaries:

- $91 \%$ of attendees at MDG meetings reported that they had developed relationships that had (or would) improved the way they care for patients;

- $67 \%$ believed that the advice they gave or received at the case conferences had (or would) helped to reduce non-elective admissions.

- Multidisciplinary learning was enabled:

- $92 \%$ of attendees at MDG meetings thought that case conferences were a good learning experience.

\section{Suggestions to improve the ONWL ICP}

A number of suggestions for improvement were made by the respondents to the surveys and during facilitated discussions at the MDG case conferences. In order to gain deeper understanding, we facilitated discussions about the issues raised with MDG chairs and members and the Integrated Management Board (comprised of director-level representatives from all partner organisations). These discussions resulted in broad agreement about nine areas to develop to improve the impact of the ONWL ICP.

- Re-visit the approach to care planning. The following suggestions about improving the care planning approach were made:

- create the care plan incrementally and opportunistically —a 40-minute care planning session is often difficult to schedule;

- make the information technology (IT) templates more integrated with the GP computer systems to use existing data from GP computer systems and to add data to those systems;

- conduct the care plan interview in a way that helps patient-led discussion on goals and actions rather than a clinician-driven action plan;

- present the care plans in a way that is practically useful to those who will use them, rather than in the way data were originally gathered-e.g. focused on contingency planning in a crisis (e.g. for carers to understand likely future scenarios, what the patient can do to self-help, contact details of other key practitioners);

- enable multiple health and social care professionals to update the care plans (e.g. secondary care clinicians prior to hospital discharge);

- prepare patients to be equal partners in the discussion by providing materials that explain what a care plan is, how they can use them and how to make the most of the discussion. Patients should be provided with copies of their care plan in language that is appropriate and relevant to their needs, and should be asked regularly for feedback both on care planning sessions and their overall care;

- make the care plans available for those other than the day-time general practice team (e.g. social care, out of hours practitioners, London Ambulance, community matrons).

- Provide a single care planning template for all patients, rather than disease-specific templates.

- Increase specificity of the guidance on social and psychological issues in pathways and templates.

- Reduce the number of hours spent in case conferences. Currently multidisciplinary meetings spend six hours each month discussing care plans, sometimes duplicating the learning. This was viewed as unsustainable in the long term.

- Continually increase the quality of the MDG meetings to enhance the collective learning and innovation, including developing the skills of the chair to lead the group, ensuring disciplined and efficient ways of presenting and discussing cases, prior planning to ensure that care plans and actions are adequately completed, and the case conferences are creatively used to stimulate learning and innovation.

- Develop new skills in partnership working. The care planning process challenges practitioners to develop new professional skills that support partnership working, for example, motivational interviewing for patients, leadership of multiprofessional teams and delivering service improvements between organisations and networks. These capabilities need to be developed through a formal training and development programme.

- Develop mechanisms to share and spread the learning beyond MDG case conferences.

- Develop an understanding of how to extend this approach to all patients at high risk, not just the diabetic and elderly.

- Integrate the ONWL ICP with other mechanisms for local service improvements. The MDG meetings could greatly increase their impact on whole system transformation by linking with the annual commissioning cycle and the annual cycle of pro- 
vider-led improvements. This would require a coordinated schedule of events at which partner organisations shape a shared agenda for whole system improvement and support shared leadership teams to lead increasingly ambitious models of integrated care. This would potentially also help to move beyond a focus on diseases and discrete improvement projects towards a broad vision for health and well-being, and multiple coordinated projects that achieve more in combination than any can on its own.

\section{Discussion}

The monthly multidisciplinary case conferences (MDG case conferences) pioneered in the first phase of the ICP (Inner North West London) have provided a mechanism that enables collaborative working between health and social care in local areas. Practitioners and patients alike find this to be a welcome development.

The second phase needs to build from this success. Participants at these case conferences need to purposefully integrate ICP activities with local programmes of work, for enhanced effects. Clinical Commissioning Groups need to stimulate local awareness of the potential of these groupings of 50,000 populationHealth Networks-to enable collaborative working over many issues to do with health improvement. Innovations supported by the Health Networks need to integrate one with another and workshop partici- pants need to sponsor such integration. Workshop chairs need to be developed as leaders of transformational change, overseeing a sequence of connected learning events within Health Networks and throughout whole boroughs, keeping partners from multiple organisations engaged in on-going interorganisational quality improvement.

\section{REFERENCES}

1 Harris M, Greaves F, Patterson S et al. The North West London Integrated Care Pilot: innovative strategies to improve care coordination for older adults and people with diabetes. Journal of Ambulatory Care Management 2012;35:216-25.

2 Steeden A. The Integrated Care Pilot in North West London. London Journal of Primary Care 2012;5:8-11 www.londonjournalofprimarycare.org.uk/articles/ 5872852.pdf

\section{ADDRESS FOR CORRESPONDENCE}

Samantha Roberts

Delivery Service Unit

North West London Cluster

15 Marylebone Road

London NW1 5JD

UK

Email: samantha.roberts@nw.london.nhs.uk

Submitted 20/1/13; comments to authors 11/3/13; revised 18/3/13 